\title{
Optical Ring Architecture Performance Evaluation using Ordinary Receiver
}

\author{
R. Udayakumar ${ }^{*}$, V. Khanaa and K. P. Kaliyamurthie ${ }^{3}$ \\ ${ }^{1}$ Associate Professor, Department of Information and Technology, Bharath University, Chennai-600073; \\ rsukumar2007@hotmail.com \\ 2Dean, Information Technology, Bharath University, Chennai-600073; drvkann62@yahoo.com \\ ${ }^{3}$ Professor \& Head, Dept. of IT, Bharath University, Chennai, India.kaliyamurthie@bharathuniv.ac.in
}

\begin{abstract}
Wavelength division multiplexing is an accepted technique to exploit the fiber bandwidth in metro network. Designing innovative architectures for metro network often means finding cost effective combinations of electronic and optic technologies and new network paradigms suited for constraints caused by available photonic components and subsystems. The three different and architectures included in this project the bulk of raw data in the optic domain while the implementation of the complex network formation is in the electronic domain. All the three ring 0 architectures perform $\lambda$ monitoring. The basic ringO architecture is based on WDM fiber ring. The three ring architectures are designed and simulated using OptSim 5.3 software.
\end{abstract}

Keywords: PON, Ring Topology, Node Architecture, Photodiode Array, LASER Array.

\section{Introduction}

Today's metropolitan area networks (MAN) are mostly synchronous optical network synchronous digital hierarchy [SONET/SDH] exhibits drawbacks, as their voice centric time division multiplexing [TDM] operation and symmetric circuit provisioning and bursting asymmetric data traffic is inefficiently supported. The SONET/SDH due to their cost significantly decreases the margins of profit in the sensitive metro market. Their inefficiencies create a severe bandwidth bottleneck at metro level called the metro gap and the wave length multiplexing [WDM] network have been investigated as solution to metro gap [10].

The passive optical network [PON] usually used as modern first mile access network. PONs can be used in specific applications in industry, business; office or army sectors as these require higher network infrastructure, together with the guarantee of maximum functionality. Therefore it is necessary to develop simple and efficient methods for protecting the critical optical units in PONs as well as of the whole optical distribution network [1].

This includes protection of an Optical Line Termination (OLT), which is a central optical unit of the whole PON. This unit provides mainly communication controlling, management and servicing functions of the whole network and it also connects the PON network into the backbone of telecommunication infrastructure, where failure or malfunction would surely result into a collapse of the whole PON. A typical optical distribution network usually has a star topology with a single branching point, or a tree topology with several branching points rendering the methods for OLT backup difficult. In case of a star or a tree topology, all optical fiber are concentrated into one single central point. The backup OLT can only be placed into the same place as the primary one. Such backup is not very reliable and the whole infrastructure is still vulnerable under many situations. Therefore ring topology is used as the optimal topology for critical application of PON [2-5].

*Corresponding author:

R. Udayakumar (rsukumar2007@hotmail.com) 
The ring topology could be easily formed by using standard passive optical splitters with symmetric or asymmetric splitting ratios. This would enable placing the backup (secondary) OLT unit into any possible position in a ring and rendering the whole infrastructure less vulnerable. The overall security of the whole infrastructure could be enhanced by its application for well protected specific situations in local area networks. Another disadvantage of PON with ring topology is its high value of insertion loss of passive optical splitters. This problem can be avoided in the optical distribution networks with ring topology formed using only standard symmetric passive splitters, resulting in an inefficient situation wherein only a limited number of Optical Network Units (ONUs) could be connected. Asymmetric passive optical splitters with splitting ratios calculated and optimized for specific scenario, could significantly balance the attenuation in the whole infrastructure, thus enabling more ONUs to be connected [8-12].

A ring topology is a network topology or circuit arrangement wherein each network device is attached along the same signal path to two other devices, resulting in a path in the shape of a ring. Each device in the network also referred to as node, handles every message that flows through the ring. Each node in the ring has a unique address. Since in a ring topology there is only one pathway between any two nodes, they are generally disrupted by the failure of a single link. The redundant topologies are utilised to eliminate network downtime caused by a single point of failure. All networks need redundancy for enhanced reliability. Network reliability is achieved through reliable equipment and network designs, tolerant to failures and faults. The FDDI networks overcome such disruption in the network by sending data on a clockwise and counter clockwise. In case there is a break in data flow, the data is wrapped back onto the complementary ring before it reaches the end of the cable. Thereby a path to every node is maintained within the complementary ring. In ring network the communication delay is directly proportional to number of nodes in the network. The bandwidth is shared on all links between devices [6].

\section{System Architecture}

\subsection{RingO Architecture}

The general architecture of the RingO network is illustrated in Figure 1. The three different versions of the network architecture preserve the same rationale and basic subsystems design [11].

The first version of the RingO network is based on a unidirectional WDM fiber ring with network nodes equipped with an interface between the electronic domain and the optical domain. The main features of the first RingO architecture are the following:

- Packet transmission is time-slotted and synchronized on all wavelengths.

- Packets have fixed length corresponding to one time slot.

- The number of nodes in the network in this first design is equal to the number of wavelengths.

- Each node is equipped with a tunable transmitter.

- Each node is able to check the state (busy/free) of all wavelengths.

\subsection{Node Architecture}

Some basic subsystems are common to all node architectures. The main functions supported by the node are the following.

1) Amplification of the optical signals in order to compensate for the losses in the node passive elements and of the downstream fiber link.

2) Demultiplexing of the WDM comb after the amplifier.

3) Monitoring the state of channels on each slot.

4) Local packet traffic generation uses a laser array driven by the node controller. The lasers are turned on for each time slot by direct current injection when a packet has to be generated. Data bits are then "written" inside the packet by an external modulator.

In order to increase the scalability of the proposed network in terms of maximum number of nodes, reduction

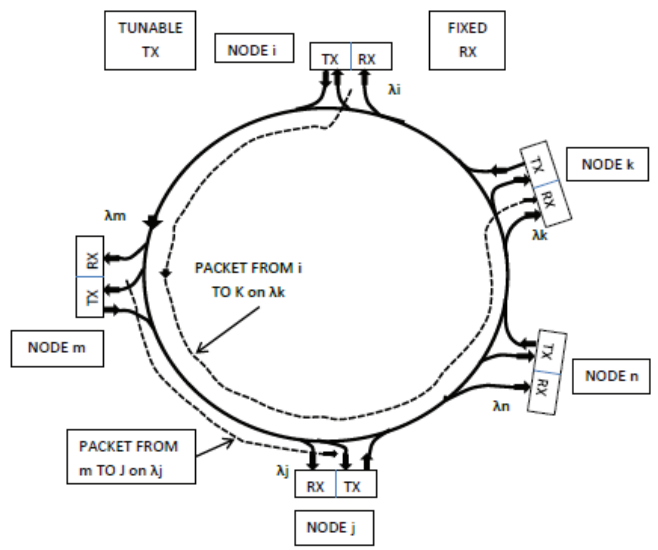

Figure 1. RingO architecture. 
of the node insertion loss, PDL, and self-filtering effects is needed. These results motivate the introduction of the second node design which is based on an add-drop filter, allowing for better cascade ability and less stringent physical constraints. An important limitation of the two previously presented RingO architectures is the fact that the number of nodes must not be greater than the number of wavelengths available on the ring. This largely impairs the scalability and the flexibility of the architecture.

The third design for RingO nodes overcomes the above limitation by means of statistically time multiplexing packets to several destinations on the same wavelength channel. This can be achieved without changing the node's hardware in a significant way: the same basic node architecture, with fast tunable transmitter and a fixed receiver can be used. The major difference of this new design is the separation between resources devoted to transmission and resources devoted to reception. The transmitted packets traverse the ring a first time and are switched to the reception path, and then received during a second ring traversal.

\section{Design and Simulation of Ringo Architecture}

The simulation block diagram designed in optsim is shown in Figure 2. In the simulation block diagram of the ringO module, it is driven by optical white Gaussian noise generator.

The noise generator has dual-sided noise spectral density of $-100 \mathrm{~dB}$. The ringO module contains the entire ring structure, shown in Figure 3.

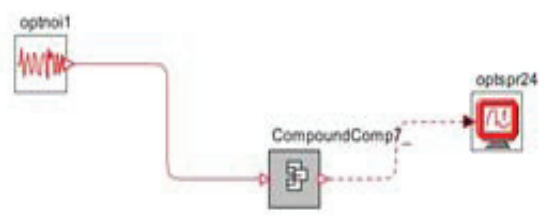

Figure 2. RingO module.

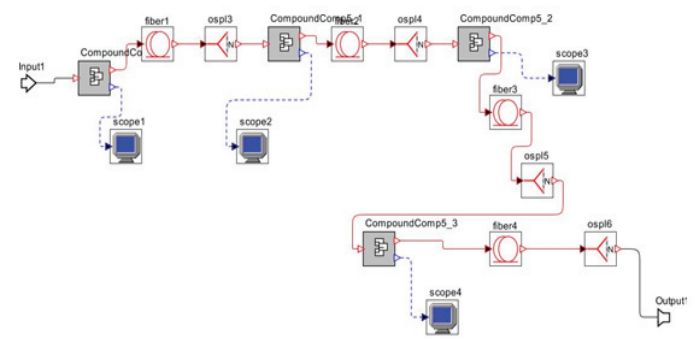

Figure 3. RingO architecture.
Here four nodes are used to form a ring structure. In this ringO architecture each node has the same structure. The nodes are designed to the wavelength $1550.00 \mathrm{~nm}$, $1550.003 \mathrm{~nm}, 1550.006 \mathrm{~nm}, 1550.009 \mathrm{~nm}$. In this ringO architecture each node is connected to the adjacent node through the optical fiber and a splitter. The fiber has length of $25 \mathrm{~km}$ and the splitter has attenuation on each output port of $-0.0 \mathrm{~dB}$.

Each node has a transmitter and a receiver. The transmitter used is tunable, consists of a data source, NRZ modulator driver, laser source, and an external modulator. The simulation block diagram in optsim is shown in Figure 4. The laser source used here is the VCSEL (Vertical Cavity Surface Emitting LASER).

The data source has a bit rate of $10 \mathrm{Gbps}$. The NRZ modulator driver has high and low signal level of $2.5 \mathrm{~V}$ and $-2.5 \mathrm{~V}$. In this design schematic the PIN diode is used for the conversion of optical signal from the noise source to the corresponding electrical signal to drive the VCSEL.

The first structure of ringO nodes consist of Erbium Doped Fiber Amplifier (EDFA) which provides a fixed gain of $10 \mathrm{~dB}$, threshold monitoring circuit, laser array etc. the threshold monitoring circuit consist of dc coupled photodiode array and a threshold comparator.

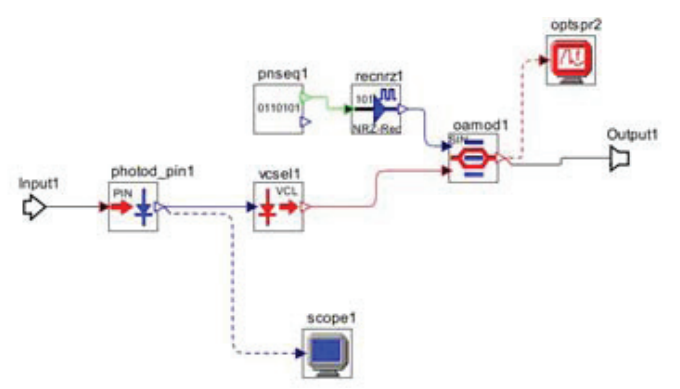

Figure 4. Arrangement of transmitter section.

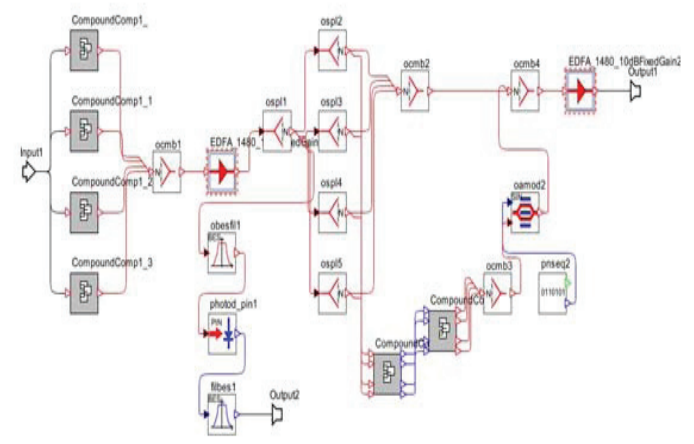

Figure 5. First structure of ringO nodes using ordinary receiver. 
The dc coupled photodiode array designed in optsim is shown in Figure 6. The photodiode array contains four PIN diodes tuned at the wavelengths $1550.00 \mathrm{~nm}, 1550.003 \mathrm{~nm}$, $1550.006 \mathrm{~nm}, 1550.009 \mathrm{~nm}$.

The laser array designed in optsim is shown in Figure 7. The laser array also contains four LASER sources each tuned at the same wavelengths as that of the PIN diodes to produce optical signal.

The performance analysis of the ringO architecture was carried out using node structures with an ordinary receiver. The ordinary receiver has a Bessel optical filter, PIN diode, and a Bessel electrical filter. The ringO architectures are the same.

The first ringO architecture contains a common structure for ringO nodes and is shown in Figure 5. In the first structure of ringO nodes the Bessel optical filter is designed as a notch filter. The PIN diode is designed to various wavelengths corresponding to four nodes in the ringO architecture. The wavelengths are $1550 \mathrm{~nm}, 1550.003 \mathrm{~nm}$, $1550.006 \mathrm{~nm}, 1550.009 \mathrm{~nm}$ respectively. The first structure of ringO nodes suffer from ASE noise [7], Polarization dependent Loss [13] and self-filtering of node passive elements

The simulation schematic of second structure of the ringO nodes is as shown in the Figure 8. It consists of the same components as in the first ringO structure. This structure has only one AWG as compared to the first structure and it uses a drop filter which gives signal to the receiver.

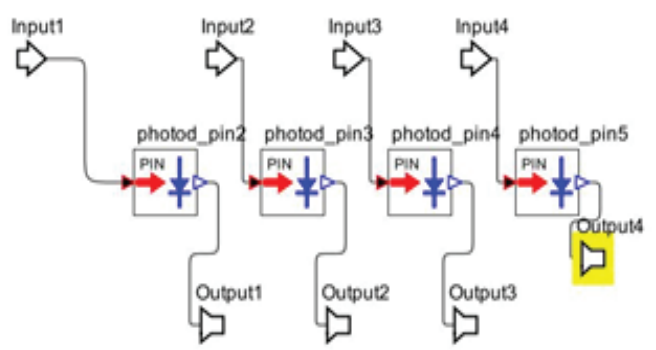

Figure 6. Arrangement of photodiode array.

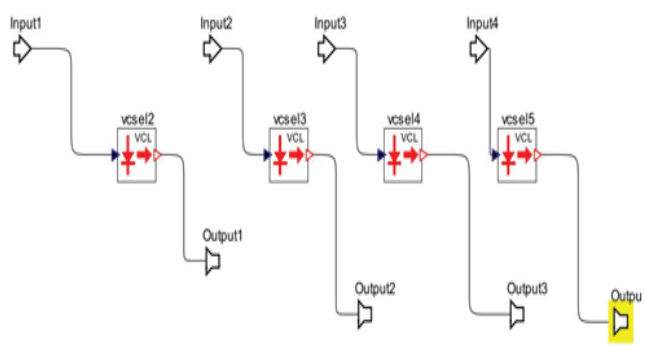

Figure 7. Arrangement of LASER array.
The first two ringO architectures have an important limitation due to the fact that the number of nodes must not be greater than the number of wavelengths available on the ring. To avoid this problem we use a third structure based on two fiber ring topology. The simulation schematic of third structure of ringO nodes is shown in Figure 9. The third structure has separate ring for transmission and reception.

\section{Results and Discussion}

The simulation is carried out for the three different ringO architectures using ordinary receiver for different bit rates such as $1 \mathrm{Gbps}$, 2Gbps, 4Gbps, $8 \mathrm{Gbps}$ and $10 \mathrm{Gbps}$. The following results are obtained.

The simulative analysis of first ringO architecture shows a gradual increase in $\mathrm{Q}$ factor with corresponding increase in bit rate. The $Q$ value increases from 9.903156dB to $11.239458 \mathrm{~dB}$ for a bit rate of $1 \mathrm{Gbps}$ to $8 \mathrm{Gbps}$. But it decreases to $8.948822 \mathrm{~dB}$ when the bit rate reaches $10 \mathrm{Gbps}$.

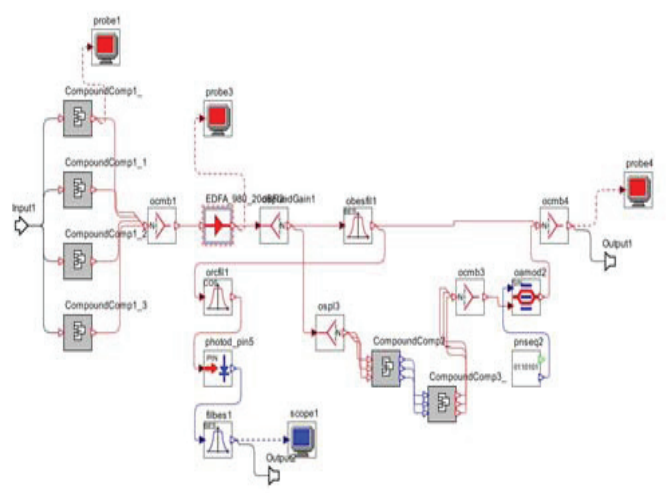

Figure 8. Second structure of ringO nodes using ordinary receiver.

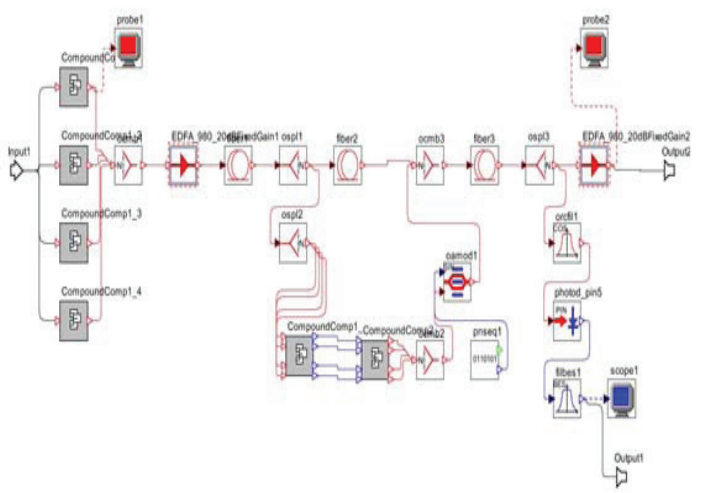

Figure 9. Third structure of ringO nodes using ordinary receiver. 
Table 1. Performance analysis of first RingO architecture

\begin{tabular}{|c|c|c|c|c|}
\hline BIT RATE & NODES & Q FACTOR & BER & JITTER \\
\hline \multirow{3}{*}{1 GBPS } & 1 & 9.903156 & 0.00106364 & 0.139172 \\
\cline { 2 - 5 } & 2 & 7.516353 & 0.00896269 & 0.118432 \\
\cline { 2 - 5 } & 3 & 10.209958 & 0.000520083 & 0.150612 \\
\cline { 2 - 5 } & 4 & 7.863007 & 0.00774782 & 0.168569 \\
\hline \multirow{3}{*}{2 GBPS } & 1 & 10.573197 & 0.00040999 & 0.139172 \\
\cline { 2 - 5 } & 2 & 7.516353 & 0.00896269 & 0.118432 \\
\cline { 2 - 5 } & 3 & 10.209958 & 0.000520083 & 0.150612 \\
\cline { 2 - 5 } & 4 & 7.863007 & 0.00774782 & 0.168569 \\
\hline \multirow{4}{*}{4 GBPS } & 1 & 10.340330 & 0.000807485 & 0.161458 \\
\cline { 2 - 5 } & 2 & 7.516353 & 0.00896269 & 0.118432 \\
\cline { 2 - 5 } & 3 & 10.209958 & 0.000520083 & 0.150612 \\
\cline { 2 - 5 } & 4 & 7.863007 & 0.00774782 & 0.168569 \\
\hline \multirow{3}{*}{8 GBPS } & 1 & 11.239458 & 0.000170171 & 0.207819 \\
\cline { 2 - 5 } & 2 & 7.516353 & 0.00896269 & 0.118432 \\
\cline { 2 - 5 } & 3 & 10.209958 & 0.000520083 & 0.150612 \\
\cline { 2 - 5 } & 4 & 7.863007 & 0.00774782 & 0.168569 \\
\hline \multirow{4}{*}{ 10GBPS } & 1 & 8.948822 & 0.00270826 & 0.0995946 \\
\cline { 2 - 5 } & 2 & 7.516353 & 0.00896269 & 0.118432 \\
\cline { 2 - 5 } & 3 & 10.209958 & 0.000520083 & 0.150612 \\
\cline { 2 - 5 } & 4 & 7.863007 & 0.00774782 & 0.168569 \\
\hline
\end{tabular}

Table 2. Performance analysis of second RingO architecture

\begin{tabular}{|c|c|c|c|c|}
\hline BIT RATE & NODES & Q FACTOR & BER & JITTER \\
\hline \multirow{3}{*}{1 GBPS } & 1 & 10.344500 & 0.000872655 & 0.125352 \\
\cline { 2 - 5 } & 2 & 12.291683 & $1.89813 \mathrm{e}^{-005}$ & 0.040529 \\
\cline { 2 - 5 } & 3 & 8.896515 & 0.00308278 & 0.248056 \\
\cline { 2 - 5 } & 4 & 10.062671 & 0.00073675 & 0.188187 \\
\hline \multirow{3}{*}{2 GBPS } & 1 & 10.344948 & 0.000871819 & 0.125352 \\
\cline { 2 - 5 } & 2 & 12.291683 & $1.89813 \mathrm{e}^{-005}$ & 0.040529 \\
\cline { 2 - 5 } & 3 & 8.896515 & 0.00308278 & 0.248056 \\
\cline { 2 - 5 } & 4 & 10.062671 & 0.00073675 & 0.188187 \\
\hline \multirow{3}{*}{ 4GBPS } & 1 & 10.348062 & 0.00087394 & 0.125352 \\
\cline { 2 - 5 } & 2 & 12.291683 & $1.89813 \mathrm{e}^{-005}$ & 0.040529 \\
\cline { 2 - 5 } & 3 & 8.896515 & 0.00308278 & 0.248056 \\
\cline { 2 - 5 } & 4 & 10.062671 & 0.00073675 & 0.188187 \\
\hline \multirow{3}{*}{ 8GBPS } & 1 & 10.349980 & 0.000869183 & 0.125352 \\
\cline { 2 - 5 } & 2 & 12.291683 & $1.89813 \mathrm{e}^{-005}$ & 0.040529 \\
\cline { 2 - 5 } & 3 & 8.896515 & 0.00308278 & 0.248056 \\
\cline { 2 - 5 } & 4 & 10.062671 & 0.00073675 & 0.188187 \\
\hline \multirow{3}{*}{ 10GBPS } & 1 & 10.341798 & 0.000865128 & 0.125352 \\
\cline { 2 - 5 } & 2 & 12.291683 & $1.89813 \mathrm{e}^{-005}$ & 0.040529 \\
\cline { 2 - 5 } & 3 & 8.896515 & 0.00308278 & 0.248056 \\
\cline { 2 - 5 } & 4 & 10.062671 & 0.00073675 & 0.188187 \\
\hline
\end{tabular}

The BER shows a variation in its values with increment in the bit rate. The jitter increases up to $8 \mathrm{Gbps}$, but it also shows a decrement when the bit rate reaches $10 \mathrm{Gbps}$. It is shown in Table 1.

The results obtained after the simulative analysis of second ring $\mathrm{O}$ architecture is shown in the Table 2.

The simulation of second ringO architecture using ordinary receiver for different bit rates shows variation in the performance parameters. The $\mathrm{Q}$ factor is 10.344500 for 1 Gbps. It increases to $10.344948 \mathrm{~dB}, 10348062 \mathrm{~dB}$, and $10.349980 \mathrm{~dB}$ for $2 \mathrm{Gbps}, 4 \mathrm{Gbps}$ and $8 \mathrm{Gbps}$ respectively. But it decreases to 10.341798 for $10 \mathrm{Gbps}$. The BER also shows irregular variation in its values.
Table 3. Performance analysis of third RingO architecture

\begin{tabular}{|c|c|c|c|c|}
\hline BIT RATE & NODES & Q FACTOR & BER & JITTER \\
\hline \multirow{3}{*}{1 GBPS } & 1 & 9.181429 & 0.00177169 & 0.187453 \\
\cline { 2 - 5 } & 2 & 10.281631 & 0.000693713 & 0.0960276 \\
\cline { 2 - 5 } & 3 & 10.746767 & 0.000291549 & 0.125684 \\
\cline { 2 - 5 } & 4 & 10.618694 & 0.000327142 & 0.199274 \\
\hline \multirow{3}{*}{ GBPS } & 1 & 9.736645 & 0.00181125 & 0.169601 \\
\cline { 2 - 5 } & 2 & 10.281631 & 0.000693713 & 0.0960276 \\
\cline { 2 - 5 } & 3 & 10.746767 & 0.000291549 & 0.125684 \\
\cline { 2 - 5 } & 4 & 10.618694 & 0.000327142 & 0.199274 \\
\hline \multirow{3}{*}{ GGBS } & 1 & 9.063712 & 0.00198887 & 0.187453 \\
\cline { 2 - 5 } & 2 & 10.281631 & 0.000693713 & 0.0960276 \\
\cline { 2 - 5 } & 3 & 10.746767 & 0.000291549 & 0.125684 \\
\cline { 2 - 5 } 8GBPS & 4 & 10.618694 & 0.000327142 & 0.199274 \\
\cline { 2 - 5 } & 1 & 9.062721 & 0.00199096 & 0.187453 \\
\cline { 2 - 5 } & 2 & 10.281631 & 0.000693713 & 0.0960276 \\
\cline { 2 - 5 } & 3 & 10.746767 & 0.000291549 & 0.125684 \\
\hline \multirow{3}{*}{10 GBPS } & 1 & 10.618694 & 0.000327142 & 0.199274 \\
\cline { 2 - 5 } & 2 & 10.28145 & 0.00198396 & 0.187453 \\
\cline { 2 - 5 } & 3 & 10.746767 & 0.000693713 & 0.0960276 \\
\cline { 2 - 5 } & 4 & 10.618694 & 0.000291549 & 0.125684 \\
\hline
\end{tabular}

The result obtained after the simulation of the third ringO architecture using ordinary receiver given in Table 3. The results obtained after the simulation of third ringO architecture shows an increase in Q value up to $2 \mathrm{Gbps}$. It is $9.181429 \mathrm{~dB}$ for $1 \mathrm{Gbps}$ and $9.736645 \mathrm{~dB}$ for $2 \mathrm{Gbps}$. The $\mathrm{Q}$ factor decreases for $4 \mathrm{Gbps}$ to $10 \mathrm{Gbps}$. The BER increases from 0.00177169 to 0.00199096 for $1 \mathrm{Gbps}$ to $8 \mathrm{Gbps}$. It decreases for $10 \mathrm{Gbps}$.

\section{Conclusion}

The ring optical network has connection to adjacent nodes and each design may vary from ring from ring. The first phase of the project was to design the three different structures of ringO nodes, various ringO architectures based on these nodes and its simulation using the software Optsim. The three structures of the ringO nodes are designed and the simulation of the first ringO node structure is performed using ordinary receiver and values for the performance parameters are obtained for the three different ringO architectures. The values of the $\mathrm{Q}$ factor show a gradual increase up to $8 \mathrm{Gbps}$ and then it decreases for $10 \mathrm{Gbps}$. The BER also increases up to $8 \mathrm{Gbps}$ and then it decreases for 10Gbps.

\section{Reference}

1. Bianciotto A, Carena A et al. Experimental WDM Packet Networks for Metro Applications: the RingO and Wonder Projects, PhotonLab and LIPAR Advanced Laboratories Dipartimento di Elettronica, Politecnico di Torino C.so Duca degli Abruzzi 24, 10129, Torino, Italy. 
2. Carena A, De Feo V et al. (2004). RingO: an Experimental WDM Optical Packet Network for Metro Applications, IEEE journal on selected areas in communications, vol 22(8), 1561-1571.

3. Turuk A K, and Kumar R (2004). A scalable and collision free Mac protocol for all optical ring network, vol 27(15), 1453-1463.

4. Su C, Chen L et al. (1997). Theory of burst-mode receiver and its applications in optical multiaccess networks, vol 15(4), 590-606.

5. Burmeister E F, Blumenthal D J et al. (2006). Optical Buffering for Next-Generation Routers, Opto-Electronics and Communications Conference.

6. Ring Network. Available From: http://en.wikipedia.org/wiki/ Ring_network

7. Encyclopaedia of Laser Physics and Technology - amplified spontaneous emission. Available From: http://www.rpphotonics.com/amplified_spontaneous_emission.html

8. Encyclopaedia of Laser physics and technology-wavelength tunability. Available From: http://www.rp-photonics.com/wavelength_tuning.html
9. Rsoft application gallery. Available From: http://www.rsoftdesign.com/products.php

10. Yang H, Herzog M et al. (2004). Metro WDM networks: performance comparison of slotted ring and AWG star networks, IEEE Journal on Selected Areas in Communications, vol 22(8), 1460-1473.

11. Georgiadis L, Guérin R et al. (1993). Throughput properties of fair policies in ring networks, IEEE/ACM Transactions on Networking, vol 1(6), 718-728.

12. Lafata P (2012). Protection of passive optical network by using ring topology, 35th International Conference on Telecommunications and Signal Processing (TSP), 105-110.

13. Gifford, and Kawachi, Polarization dependent loss measurement of passive optical components - application note.

14. Carter B (2001). Using Texas instruments spice models in PSpice, Application Report, SLOA070, Texas Instruments, Dallas, TX. University of Pennsylvania Department of Electrical and Systems Engineering PSPICEA brief primer. 